\title{
Synthesis and Properties of Mixed Ligand Complexes of Diorganotin (IV): Part (VI): Synthesis and Characterisation of Antibacterial Benzoylacetonato Diorganotin (IV)-O-Alkyl Trithiophosphates
}

\author{
Leena Chordia and Alok Chaturvedi* \\ Department of Chemistry, Government College, Ajmer - 305001, India \\ E-mail:alok_chat.ajm@rediffmail.com; leenachordia@rediffinail.com
}

\begin{abstract}
The reaction of diorganotin dichloride with benzoyl acetone and O-alkyl trithiophosphate have been carried out in 1:1:1 molar ratio in refluxing benzene. The newly synthesized complexes $\mathrm{R}_{2} \mathrm{Sn}[\mathrm{PhCOCHCOMe}]\left[\mathrm{SSH}(\mathrm{S}) \mathrm{POR}^{\prime}\right]$ are found to be non volatile, light yellow coloured crystalline solids, monomeric in nature and highly sensitive towards atmospheric moisture. These complexes were characterized by elemental analysis (S,Sn), molecular weight measurements, IR and NMR $\left({ }^{1} \mathrm{H},{ }^{31} \mathrm{P},{ }^{119} \mathrm{Sn}\right)$ spectral data. On the basis of spectral studies, the bidentate nature of benzoyl acetonate and O-alkyl trithiophosphate moieties and the hexacoordinated nature of tin atom was established. All these newly synthesized compounds were tested for their antibacterial activity against gram positive and gram negative bacteria. All compounds showed good activity against bacteria.
\end{abstract}

Key words: Diorganotin dichloride, benzoyl acetone, O-alkyl trithiophosphates.

\section{INTRODUCTION}

In continuation of our earlier investigations on mixed ligand complexes of diorganotin(IV) $/ 1-4 /$, the work has been extended to investigate the effect of $\mathrm{O}$-alkyl trithiophosphates on the bonding modes of $\beta$ diketonate moiety towards the central tin atom. O-alkyl trithiophosphates contribute an important series of ligands which show an interesting versatility in their chemical bonding modes (e.g. bidentate, chelating / bridging) towards different metals $/ 5 /$.

The chemistry of metal $\beta$-diketonate has been extensively reported in the literature $/ 6,7 /$. The mixed derivatives of $\beta$-diketonate with alkoxides $/ 8 /$, chlorides $/ 9 /$, acetates $/ 10 /$ and alkylxanthates $/ 11 /$ have been reported in the literature. The mixed derivatives of the type $\mathrm{R}_{2} \mathrm{Sn}\left[\mathrm{OCOCH} \mathrm{CO}_{3}\right]\left[\mathrm{S}_{2} \mathrm{P}(\mathrm{OR})_{2}\right]$ have been reported from our laboratory $/ 4$ / in which monofunctional bidentate behaviour of the ligand has been established. In 
general, $\beta$-diketonates show facile reactivity towards metal alkoxide, in which alkoxy group of metal alkoxide is easily replaced by the enolate form of $\beta$-diketonate. Diisopropyltin(IV) trithiophosphate and their adducts with nitrogen donar bases have also been reported in the literature /12/. O-alkyl, O-cycloalkyl and O-aryl trithiophosphato derivatives of trimethyl- and tributyl tin(IV) chloride have also been synthesized and characterized by various spectral studies $/ 13 /$.

The mixed derivatives of diorganotin (IV) with $\beta$-diketonates and alkyl trithiophosphates have not been studied so far. In view of the above, it was considered of interest to synthesize and study the chemical bonding modes in these new derivatives. These newly synthesized derivatives showed higher anti-bacterial activity at $500 \mu \mathrm{g} / \mathrm{mL}$ concentration against gram-negative and gram-positive bacteria.

In the present communication, we report the synthesis and spectroscopic properties of mixed ligand complexes of the type $\mathrm{R}_{2} \mathrm{Sn}\left[\mathrm{SSH}(\mathrm{S}) \mathrm{POR}^{\prime}\right]\left[\mathrm{C}_{6} \mathrm{H}_{5} \mathrm{COCHCOCH}_{3}\right]$.

\section{MATERIALS AND METHODS}

Owing to the hygroscopic nature of the mixed ligand complexes, stringent precautions were taken to exclude moisture. Sulphur and tin were estimated gravimetrically as barium sulphate (Messenger's method /14/) and tin oxide, respectively. A Knauer vapour pressure osmometer in chloroform was used for molecular weight determination. FTIR spectra were recorded on a Shimadzu $8201 \mathrm{PC}$ spectrophotometer in the range of 4000-200 $\mathrm{cm}^{-1}$ using CsI cells. ${ }^{1} \mathrm{H}$ NMR spectra (in $\mathrm{CDCl}_{3}$ ) and ${ }^{31} \mathrm{P}$ and ${ }^{119} \mathrm{Sn}$ NMR spectra (in benzene) were recorded on Bruker DRX-300 spectrophotometer using TMS (for ${ }^{1} \mathrm{H}$ ), $\mathrm{H}_{3} \mathrm{PO}_{4}$ (for ${ }^{31} \mathrm{P}$ ) and $\mathrm{Me}_{4} \mathrm{Sn}$ (for ${ }^{119} \mathrm{Sn}$ ) as external references.

\section{Synthesis of $\mathrm{Me}_{2} \mathrm{Sn}$ [PhCOCHCOMe][SSH(S)POPh]}

A mixture of dimethyltin dichloride (1.08 g; $4.91 \mathrm{mmole})$, benzoyl acetone ( $0.79 \mathrm{~g} ; 4.88 \mathrm{mmole})$, Ophenyl trithiophosphate $(1.46 \mathrm{~g} ; 4.90 \mathrm{mmole})$ are taken in dry benzene $(\sim 35 \mathrm{~mL})$. The reaction mixture is then refluxed for about 10 hours, Precipitate of $\mathrm{KCl}$ formed during the course of the reaction, is removed by filteration in anhydrous condition and excess of solvent (benzene) is removed under reduced pressure. A light yellow coloured crystalline solid $\mathrm{Me}_{2} \mathrm{Sn}$ [PhCOCHCOMe][SSH(S)POPh] is obtained, $2.46 \mathrm{~g}$ yield; $95.35 \%$. Calcd. for $\mathrm{Me}_{2} \mathrm{Sn}[\mathrm{PhCOCHCOMe}][\mathrm{SSH}(\mathrm{S}) \mathrm{POPh}], \mathrm{S}=18.08 \%, \mathrm{Sn}=22.41 \%$; Found: $\mathrm{S}=17.95 \%$, $\mathrm{Sn}=$ $21.26 \%$.

Details of similar derivatives are also summarized in Table-I.

\section{RESULTS AND DISCUSSION}

Diorganotin dichloride, benzoyl acetone and dipotassium salt of O-alkyl trithiophosphate are mixed in stoichiometric ratio and refluxed in dry benzene for $\sim 10$ hours. Benzoylacetonato diorganotin(IV)-O-alkyl trithiophosphate is formed. The $\mathrm{KCl}$ formed is being removed by alkoxy funnel in anhydrous condition. 
$\mathrm{R}_{2} \mathrm{SnCl}_{2}+\mathrm{C}_{6} \mathrm{H}_{5} \mathrm{COCH}_{2} \mathrm{COCH}_{3}+\mathrm{R}^{\prime} \mathrm{OPS}_{3} \mathrm{~K}_{2} \underset{\text { dry benzene }}{10 \text { hours }} \rightarrow$

$\left[\mathrm{R}=\mathrm{Me}, \mathrm{Bu}, \mathrm{Ph} ; \mathrm{R}^{\prime}=\mathrm{Me}, \mathrm{Et}, \mathrm{Pr}^{\mathrm{i}}, \mathrm{Bu}^{\mathrm{i}}, \mathrm{Ph}\right]$

$\mathrm{R}_{2} \mathrm{Sn}\left[\mathrm{SSH}(\mathrm{S}) \mathrm{POR}^{\prime}\right]\left[\mathrm{C}_{6} \mathrm{H}_{5} \mathrm{COCHCOCH}_{3}\right]$

The complexes thus formed are light-yellow coloured crystalline solids, soluble in common organic solvents, monomeric in nature and are extremely sensitive towards atmospheric moisture.

\section{SPECTRAL STUDIES}

\section{IR Spectra :}

IR spectra of these complexes have been recorded in the region $4000-200 \mathrm{~cm}^{-1} \quad$ (Table-II). A comparison of the IR spectra of these complexes [benzoylacetonato diorganotin(lV)-O-alkyl trithiophosphates] with those of the starting materials is as follows:

(1) A new absorption band in the region $365.8-392.4 \mathrm{~cm}^{-1}$ has been assigned to vSn-S linkage.

(2) Appearance of a new sharp band at $442.5-458.3 \mathrm{~cm}^{-1}$, assigned to $v \mathrm{Sn}-\mathrm{O}$ linkage.

(3) The strong absorption bands in the region between $1428.3-1474.5 \mathrm{~cm}^{-1}$ and $1567.6-1598.7 \mathrm{~cm}^{-1}$ have been assigned to coordinated $v \mathrm{C}=\mathrm{O}$ and $v \mathrm{C}=\mathrm{C}$ stretching vibrations, respectively.

(4) A new broad absorption band is observed in the region $2351.2-2382.5 \mathrm{~cm}^{-1}$. This band indicates the presence of a S-H bond in the complexes.

(5) No absorption band is observed at $1100-1200 \mathrm{~cm}^{-1}$, which indicates that $\mathrm{P}=\mathrm{O}$ linkage is absent in these newly synthesized compounds and also indicates that the O-alkyl trithiophosphate moiety, in these newly synthesized compounds is present in thiono form, not in thiolo form.

(6) In these complexes, vP-S absorption band is shifted towards lower frequency, which indicates that this group is strongly chelated with the central tin atom.

\section{NMR Spectra}

${ }^{1} \mathbf{H}$ :

The PMR spectra of these complexes show characteristic resonance signals for benzoylacetonato moiety, alkyl and aryl protons present on tin atom and O-alkyl protons present on phosphorus atom (Table-III). A singlet at $\sim \delta 2.6 \mathrm{ppm}$ is observed which is due to O-methoxy protons of O-alkyl trithiophosphate moiety in these complexes. 


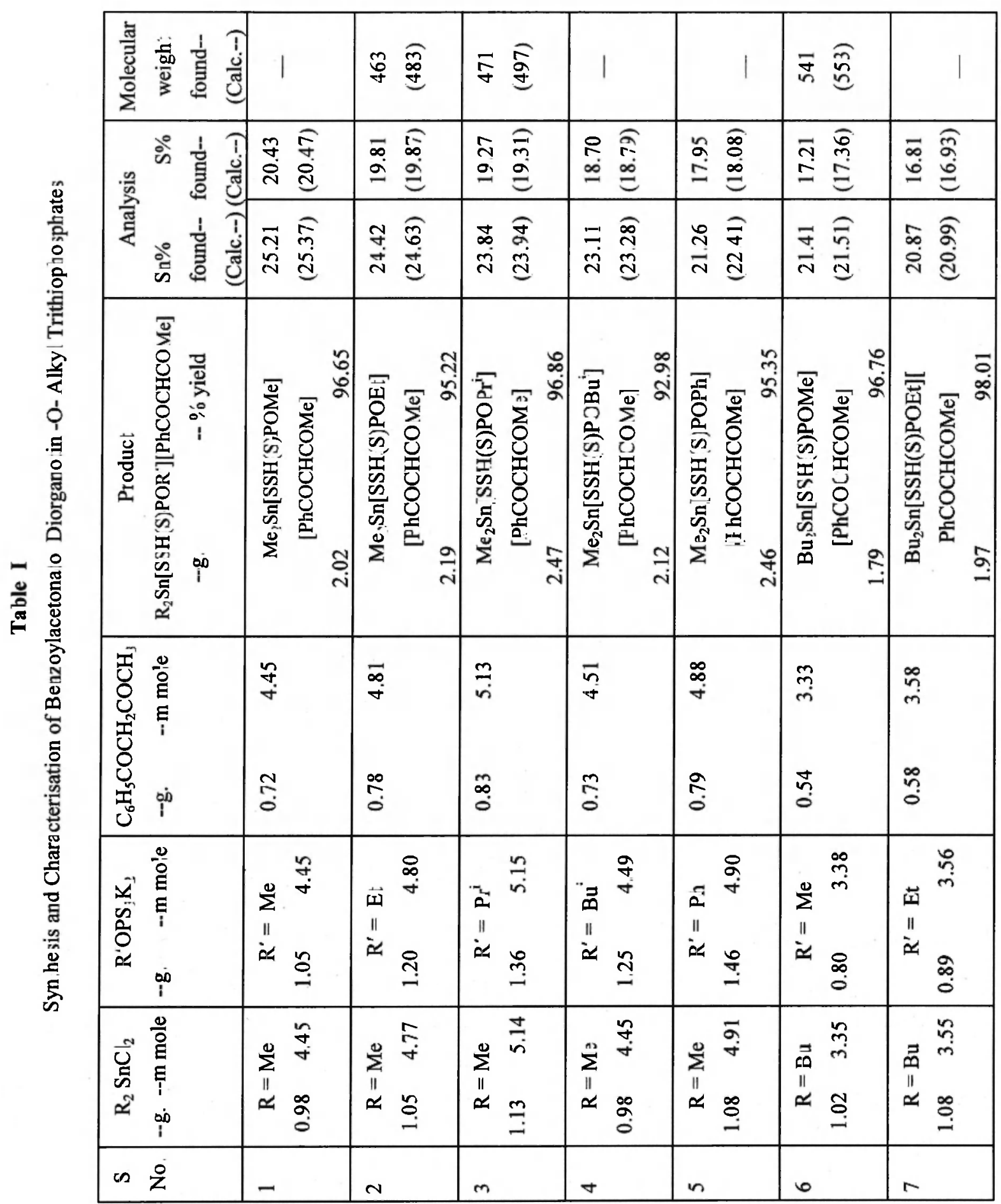




\begin{tabular}{|c|c|c|c|c|c|c|c|}
\hline 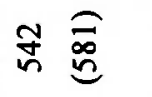 & 1 & I & | & 䓀 & 1 & 1 & 亏 \\
\hline 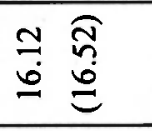 & 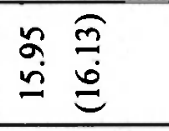 & 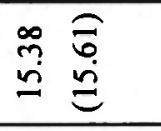 & $\begin{array}{l}\bar{\sigma} \\
\dot{0} \\
\underline{0}\end{array}$ & 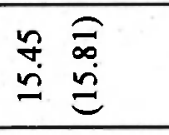 & 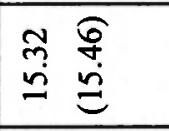 & 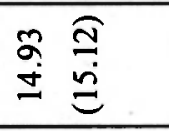 & 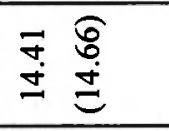 \\
\hline 守 & 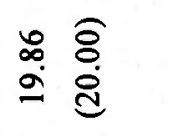 & $\begin{array}{l}\overline{\tilde{a}} \\
\stackrel{2}{2}\end{array}$ & 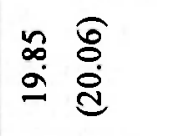 & 囟 & 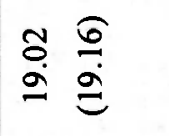 & 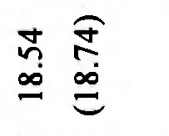 & $\stackrel{\overbrace{}}{\stackrel{E}{\Xi}}$ \\
\hline 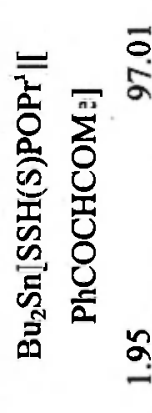 & 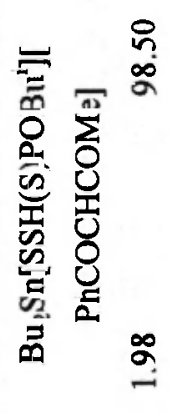 & 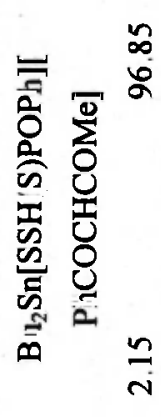 & 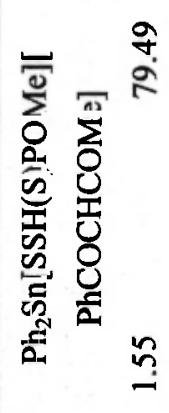 & 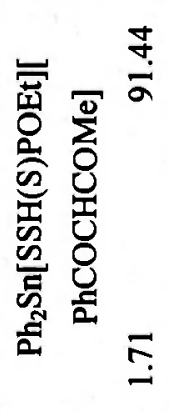 & 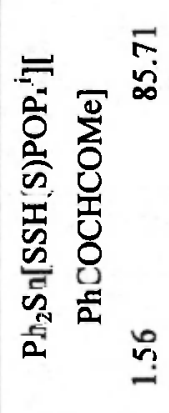 & 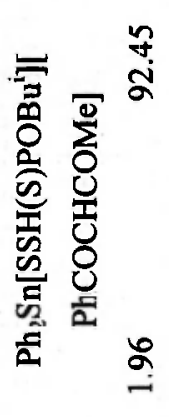 & 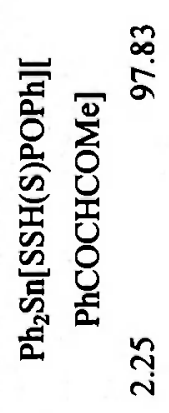 \\
\hline$\stackrel{+}{\stackrel{0}{0}}$ & $\stackrel{\vec{m}}{\mathrm{~m}}$ & $\underset{\sim}{\Delta}$ & $\stackrel{m}{\tilde{m}}$ & $\stackrel{\infty}{\circ}$ & ๙̊ & $\underset{j}{\vec{m}}$ & $\underset{\sim}{\tilde{n}}$ \\
\hline ڤొ & $\tilde{n}$ & ڤ̂े & ț & ํํㅁ & $\stackrel{\infty}{0}$ & $\tilde{n}$ & ñ \\
\hline 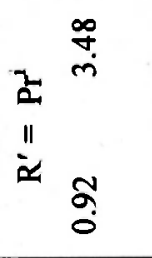 & 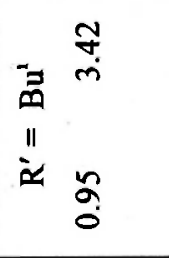 & 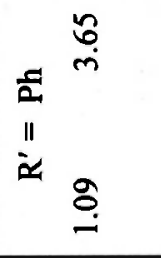 & 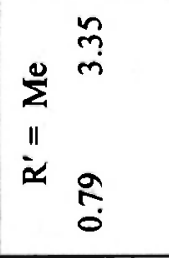 & 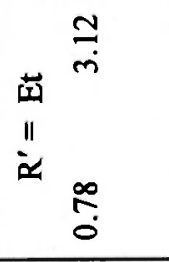 & 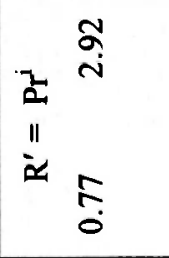 & 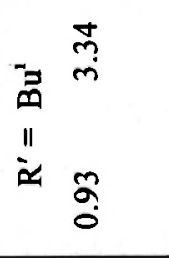 & 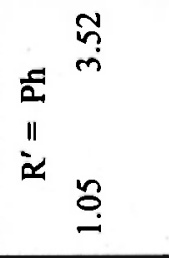 \\
\hline 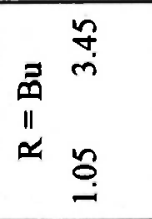 & 岧 & 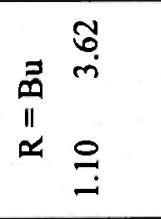 & 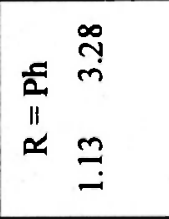 & 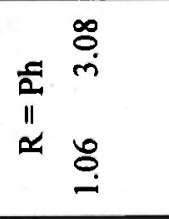 & 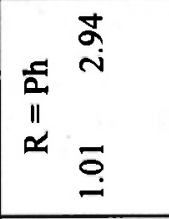 & 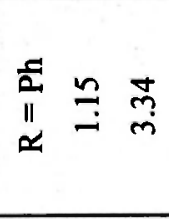 & 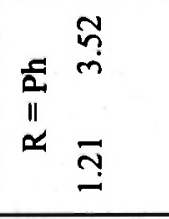 \\
\hline & & $\subseteq$ & & & & & \\
\hline
\end{tabular}




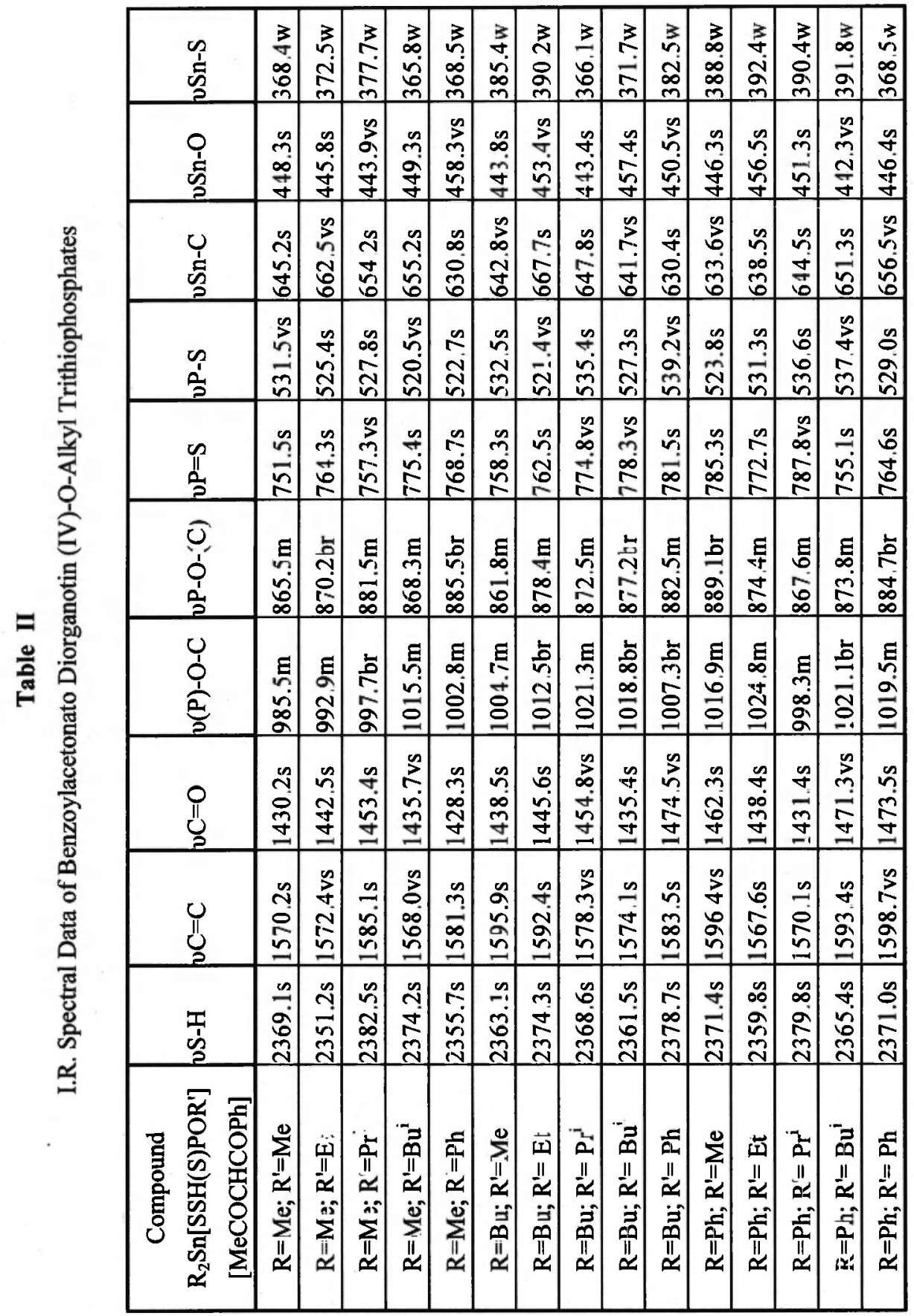




\section{Table III}

PMR Spectral Data of Benzoylacetonato Diorganotin(IV)-O-Alkyl Trithiophosphates

\begin{tabular}{|c|c|c|}
\hline S.N & C & m) \\
\hline 1. & $\begin{array}{l}\mathrm{Me}_{2} \mathrm{Sn}[\mathrm{SSH}(\mathrm{S}) \mathrm{POMe}] \\
{\left[\mathrm{C}_{6} \mathrm{H}_{5} \mathrm{COCHCOCH}\right]}\end{array}$ & $\begin{array}{l}1.53, \mathrm{~s}, 6 \mathrm{H}\left(\mathrm{Sn}-\mathrm{CH}_{3}\right) ; 2.05, \mathrm{~s}, 3 \mathrm{H}\left(\mathrm{CH}_{3}\right) ; 2.45, \mathrm{~s}, 3 \mathrm{H}\left(\mathrm{OCH}_{3}\right) \\
3.42, \mathrm{~s}, 1 \mathrm{H}(\mathrm{SH}) ; 5.22, \mathrm{~s}, 1 \mathrm{H}(\mathrm{CH}) ; 7.25-7.38, \mathrm{~m}, 5 \mathrm{H}\left(\mathrm{C}_{6} \mathrm{H}_{5}\right)\end{array}$ \\
\hline 2 & $\begin{array}{l}\mathrm{Me}_{2} \mathrm{Sn}[\mathrm{SSH}(\mathrm{S}) \mathrm{POEt}] \\
{\left[\mathrm{C}_{6} \mathrm{H}_{5} \mathrm{COCHCOCH}{ }_{3}\right]}\end{array}$ & $\begin{array}{l}1.64, \mathrm{~s}, 6 \mathrm{H}\left(\mathrm{Sn}-\mathrm{CH}_{3}\right) ; 2.11, \mathrm{~s}, 3 \mathrm{H}\left(\mathrm{CH}_{3}\right) ; 2.24-2.38, \mathrm{t}, \quad 3 \mathrm{H}\left(\mathrm{CH}_{3}\right) ; 2.52-2.67, \quad q, \\
2 \mathrm{H}\left(\mathrm{OCH}_{2}\right) ; 3.51, \mathrm{~s}, 1 \mathrm{H}(\mathrm{SH}) ; 5.24, \mathrm{~s}, 1 \mathrm{H}(\mathrm{CH}) ; 7.28-7.41, \mathrm{~m}, 5 \mathrm{H}\left(\mathrm{C}_{6} \mathrm{H}_{5}\right)\end{array}$ \\
\hline 3 & $\begin{array}{l}\mathrm{Me}_{2} \mathrm{Sn}\left[\mathrm{SSH}(\mathrm{S}) \mathrm{POPr}^{\mathrm{i}}\right] \\
{\left[\mathrm{C}_{6} \mathrm{H}_{5} \mathrm{COCHCOCH}\right]}\end{array}$ & $\begin{array}{l}2.02, \mathrm{~s}, 9 \mathrm{H}\left(\mathrm{Sn}-\mathrm{CH}_{3}, \mathrm{CH}_{3}\right) ; 2.25-2.32, \mathrm{~d}, 6 \mathrm{H}\left(\mathrm{CH}_{3}-\mathrm{Pr}^{\mathrm{i}}\right) ; 2.65-2.81, \mathrm{~m}, 1 \mathrm{H}(\mathrm{OCH}) \\
3.38, \mathrm{~s}, 1 \mathrm{H}(\mathrm{SH}) ; 5.13, \mathrm{~s}, 1 \mathrm{H}(\mathrm{CH}) ; 7.34-7.47, \mathrm{~m}, 5 \mathrm{H}\left(\mathrm{C}_{6} \mathrm{H}_{5}\right)\end{array}$ \\
\hline 4 & $\begin{array}{l}\mathrm{Me}_{2} \mathrm{Sn}\left[\mathrm{SSH}(\mathrm{S}) \mathrm{POBu}^{\mathrm{i}}\right] \\
{\left[\mathrm{C}_{6} \mathrm{H}_{5} \mathrm{COCHCOCH}{ }_{3}\right]}\end{array}$ & $\begin{array}{l}2.01, \mathrm{~s}, 6 \mathrm{H}\left(\mathrm{Sn}-\mathrm{CH}_{3}\right) ; 2.25, \mathrm{~s}, 3 \mathrm{H}\left(\mathrm{CH}_{3}\right) ; 2.54-2.61, \mathrm{~d}, 6 \mathrm{H}\left(\mathrm{CH}_{3}-\mathrm{Bu}^{\mathrm{i}}\right) ; 3.15, \mathrm{~s}, \\
1 \mathrm{H}(\mathrm{SH}) ; 3.84-3.95, \mathrm{~d}, 2 \mathrm{H}\left(\mathrm{OCH}_{2}\right) ; 4.61-4.78, \mathrm{~m}, 1 \mathrm{H}\left(\mathrm{CH}^{\mathrm{i}} \mathrm{Bu}^{\mathrm{i}}\right) ; 5.22, \mathrm{~s}, \\
1 \mathrm{H}(\mathrm{CH}) ; 7.21-7.42, \mathrm{~m}, 5 \mathrm{H}\left(\mathrm{C}_{6} \mathrm{H}_{5}\right)\end{array}$ \\
\hline 5 & $\begin{array}{l}\mathrm{Me}_{2} \mathrm{Sn}[\mathrm{SSH}(\mathrm{S}) \mathrm{POPh}] \\
{\left[\mathrm{C}_{6} \mathrm{H}_{5} \mathrm{COCHCOCH} \mathrm{H}_{3}\right]}\end{array}$ & $\begin{array}{l}2.24, \mathrm{~s}, 9 \mathrm{H}\left(\mathrm{Sn}-\mathrm{CH}_{3}, \mathrm{CH}_{3}\right) ; 3.51, \mathrm{~s}, 1 \mathrm{H}(\mathrm{SH}) ; 5.45, \mathrm{~s}, 1 \mathrm{H}(\mathrm{CH}) ; 7.12-7.30, \mathrm{~m}, \\
5 \mathrm{H}\left(\mathrm{C}_{6} \mathrm{H}_{5}\right) ; 7.51-7,68, \mathrm{~m}, 5 \mathrm{H}\left(\mathrm{OC}_{6} \mathrm{H}_{5}\right)\end{array}$ \\
\hline 6 & $\begin{array}{l}\mathrm{Bu}_{2} \mathrm{Sn}[\mathrm{SSH}(\mathrm{S}) \mathrm{POMe}] \\
{\left[\mathrm{C}_{6} \mathrm{H}_{5} \mathrm{COCHCOCH} \mathrm{CH}_{3}\right]}\end{array}$ & $\begin{array}{l}0.82-0.96, \mathrm{t}, 6 \mathrm{H}\left(\mathrm{CH}_{3}\right) ; 1.56-1.68, \mathrm{~m}, 12 \mathrm{H}\left(\mathrm{Sn}-\left(\mathrm{CH}_{2}\right)_{3}\right) ; 1.98, \mathrm{~s}, 3 \mathrm{H}\left(\mathrm{CH}_{3}\right) ; 2.35, \mathrm{~s}, \\
3 \mathrm{H}\left(\mathrm{OCH}_{3}\right) ; 3.51 . \mathrm{s}, 1 \mathrm{H}(\mathrm{SH}) ; 5.12, \mathrm{~s}, 1 \mathrm{H}(\mathrm{CH}) ; 7.18-7.32, \mathrm{~m}, 5 \mathrm{H}\left(\mathrm{C}_{6} \mathrm{H}_{5}\right)\end{array}$ \\
\hline 7 & $\begin{array}{l}\text { (S)POEt] } \\
{\left[\mathrm{COCH}_{3}\right]}\end{array}$ & $\begin{array}{l}0.85-0.96, \mathrm{t}, 6 \mathrm{H}\left(\mathrm{CH}_{3}\right) ; 1.45-1.57, \mathrm{~m}, 12 \mathrm{H}\left(\mathrm{Sn}-\left(\mathrm{CH}_{2}\right)_{3}\right) ; 2.02, \mathrm{~s}, 3 \mathrm{H}\left(\mathrm{CH}_{3}\right) ; 2.32- \\
2.43, \mathrm{t}, 3 \mathrm{H}\left(\mathrm{CH}_{3}\right) ; 2.81-2.97, \mathrm{q}, 2 \mathrm{H}\left(\mathrm{OCH}_{2}\right) ; 3.41, \mathrm{~s}, 1 \mathrm{H}(\mathrm{SH}) ; 5.23, \mathrm{~s}, 1 \mathrm{H}(\mathrm{CH}) ; \\
7.31-7.45, \mathrm{~m}, 5 \mathrm{H}\left(\mathrm{C}_{6} \mathrm{H}_{5}\right)\end{array}$ \\
\hline 8 & $\begin{array}{l}\mathrm{Bu}_{2} \mathrm{Sn}\left[\mathrm{SSH}(\mathrm{S}) \mathrm{POPr}^{\mathrm{i}}\right] \\
{\left[\mathrm{C}_{6} \mathrm{H}_{5} \mathrm{COCHCOCH} 3\right]}\end{array}$ & $\begin{array}{l}0.81-0.89, \mathrm{t}, 6 \mathrm{H}\left(\mathrm{CH}_{3}\right) ; 1.52-1.63, \mathrm{~m}, 12 \mathrm{H}\left(\mathrm{Sn}-\left(\mathrm{CH}_{2}\right)_{3}\right) ; 1.95, \mathrm{~s}, 3 \mathrm{H}\left(\mathrm{CH}_{3}\right) ; 2.14- \\
2.21, \mathrm{~d}, 6 \mathrm{H}\left(\mathrm{CH}_{3}-\mathrm{Pr}^{\mathrm{l}}\right) ; 3.35, \mathrm{~s}, 1 \mathrm{H}(\mathrm{SH}) ; 3.61-3.77, \mathrm{~m}, 1 \mathrm{H}\left(\mathrm{OCH}-\mathrm{Pr}^{i}\right) ; 5.42, \mathrm{~s} \\
1 \mathrm{H}(\mathrm{CH}) ; 7.24-7.38, \mathrm{~m}, 5 \mathrm{H}\left(\mathrm{C}_{6} \mathrm{H}_{5}\right)\end{array}$ \\
\hline 9 & $\begin{array}{l}\left.\mathrm{OBu}^{\mathrm{l}}\right] \\
\left.\mathrm{CH}_{3}\right]\end{array}$ & $\begin{array}{l}0.84-0.97, \mathrm{t}, 6 \mathrm{H}\left(\mathrm{CH}_{3}\right) ; 1.49-1.62, \mathrm{~m}, 12 \mathrm{H}\left(\mathrm{Sn}-\left(\mathrm{CH}_{2}\right)_{3}\right) ; 1.98, \mathrm{~s}, 3 \mathrm{H}\left(\mathrm{CH}_{3}\right) ; 2.28- \\
2.39, \mathrm{~d}, 6 \mathrm{H}\left(\mathrm{CH}_{3}-\mathrm{Bu}^{\mathrm{l}}\right) ; 3.26, \mathrm{~s}, 1 \mathrm{H}(\mathrm{SH}) ; 3.81-3.92, \mathrm{~d}, 2 \mathrm{H}\left(\mathrm{OCH}_{2}\right) ; 4.72-4.91, \mathrm{~m}, \\
1 \mathrm{H}\left(\mathrm{CH}-\mathrm{Bu}^{\mathrm{i}}\right) ; 5.32, \mathrm{~s}, 1 \mathrm{H}(\mathrm{CH}) ; 7.34-7.47, \mathrm{~m}, 5 \mathrm{H}\left(\mathrm{C}_{6} \mathrm{H}_{5}\right)\end{array}$ \\
\hline 10 & $\mathrm{OPh}$ & $\begin{array}{l}0.85-1.02, \mathrm{t}, 6 \mathrm{H}\left(\mathrm{CH}_{3}\right) ; 1.64-1.78, \mathrm{~m}, 12 \mathrm{H}\left(\mathrm{Sn}-\left(\mathrm{CH}_{2}\right)_{3}\right) ; 1.87, \mathrm{~s}, 3 \mathrm{H}\left(\mathrm{CH}_{3}\right) ; 3.52 \\
\text { s, } 1 \mathrm{H}(\mathrm{SH}) ; 5.39, \mathrm{~s}, 1 \mathrm{H}(\mathrm{CH}) ; 7.31-7.44, \mathrm{~m}, 5 \mathrm{H} \quad\left(\mathrm{C}_{6} \mathrm{H}_{5}\right) ; 7.84-7.97, \mathrm{~m}, \\
5 \mathrm{H}\left(\mathrm{OC}_{6} \mathrm{H}_{5}\right)\end{array}$ \\
\hline 11 & $\begin{array}{l}\text { S)POMe] } \\
\left.\mathrm{ICOCH}_{3}\right]\end{array}$ & $\begin{array}{l}2.22, \overline{\mathrm{s}}, 3 \mathrm{H}\left(\mathrm{CH}_{3}\right) ; 3.11, \mathrm{~s}, 3 \mathrm{H}\left(\mathrm{OCH}_{3}\right) ; 3.34, \mathrm{~s}, 1 \mathrm{H}(\mathrm{SH}) \\
7.52, \mathrm{~m}, 15 \mathrm{H}\left(\mathrm{C}_{6} \mathrm{H}_{5}\right)\end{array}$ \\
\hline 12 & $\begin{array}{l}\mathrm{Ph}_{2} \mathrm{Sn}[\mathrm{SSH}(\mathrm{S}) \mathrm{POEt}] \\
{\left[\mathrm{C}_{6} \mathrm{H}_{5} \mathrm{COCHCOCH}\right]}\end{array}$ & $\begin{array}{l}2.33, \mathrm{~s}, 3 \mathrm{H}\left(\mathrm{CH}_{3}\right) ; 2.51, \mathrm{t}, 3 \mathrm{H}\left(\mathrm{CH}_{3}\right) ; 2.72-2.84, \mathrm{q}, 2 \mathrm{H}\left(\mathrm{OCH}_{2}\right) ; 3.47, \mathrm{~s}, 1 \mathrm{H}(\mathrm{SH}) \\
5.41, \mathrm{~s}, 1 \mathrm{H}(\mathrm{CH}) ; 7.42-7.58, \mathrm{~m}, 15 \mathrm{H}\left(\mathrm{C}_{6} \mathrm{H}_{5}\right)\end{array}$ \\
\hline 13 & $\begin{array}{l}\mathrm{Ph}_{2} \mathrm{Sn}\left[\mathrm{SSH}(\mathrm{S}) \mathrm{POPr}^{\mathrm{i}}\right] \\
{\left[\mathrm{C}_{6} \mathrm{H}_{5} \mathrm{COCHCOCH}{ }_{3}\right]}\end{array}$ & $\begin{array}{l}2.13, \mathrm{~s}, 3 \mathrm{H}\left(\mathrm{CH}_{3}\right) ; 2.24-2.32, \mathrm{~d}, 6 \mathrm{H}\left(\mathrm{CH}_{3}-\mathrm{Pr}^{\mathrm{i}}\right) ; 2.91-3.03, \mathrm{~m}, 1 \mathrm{H}(\mathrm{OCH}) ; 3.48, \mathrm{~s} \\
1 \mathrm{H}(\mathrm{SH}) ; 5.27, \mathrm{~s}, 1 \mathrm{H}(\mathrm{CH}) ; 7.37-7.52, \mathrm{~m}, 15 \mathrm{H}\left(\mathrm{C}_{6} \mathrm{H}_{5}\right)\end{array}$ \\
\hline 14 & $\begin{array}{l}\mathrm{Ph}_{2} \mathrm{Sn}\left[\mathrm{SSH}(\mathrm{S}) \mathrm{POBu}^{\mathrm{i}}\right] \\
{\left[\mathrm{C}_{6} \mathrm{H}_{5} \mathrm{COCHCOCH}{ }_{3}\right]}\end{array}$ & $\begin{array}{l}2.18, \mathrm{~s}, 3 \mathrm{H}\left(\mathrm{CH}_{3}\right) ; 2.24-2.46, \mathrm{~d}, 6 \mathrm{H}\left(\mathrm{CH}_{3}-\mathrm{Bu}^{\prime}\right) ; 3.27, \mathrm{~s}, 1 \mathrm{H}(\mathrm{SH}) ; 4.21-4.45, \quad \mathrm{~m}, \\
1 \mathrm{H}\left(\mathrm{CH}-\mathrm{Bu}^{\mathrm{i}}\right) ; 5.22-5.31, \mathrm{~d}, 2 \mathrm{H}\left(\mathrm{OCH}_{2}\right) 5.64, \mathrm{~s}, 1 \mathrm{H}(\mathrm{CH}) ; 7.24-7.43, \mathrm{~m}, 15 \mathrm{H}\left(\mathrm{C}_{6} \mathrm{H}_{5}\right)\end{array}$ \\
\hline 15 & $\begin{array}{l}\mathrm{Ph}_{2} \mathrm{Sn}[\mathrm{SSH}(\mathrm{S}) \mathrm{POPh}] \\
{\left[\mathrm{C}_{6} \mathrm{H}_{5} \mathrm{COCHCOCH}{ }_{3}\right]}\end{array}$ & $\begin{array}{l}2.24, \mathrm{~s}, 3 \mathrm{H}\left(\mathrm{CH}_{3}\right) ; 3.45, \mathrm{~s}, 1 \mathrm{H}(\mathrm{SH}) ; 5.52, \mathrm{~s}, 1 \mathrm{H}(\mathrm{CH}) ; 7.28-7.46, \mathrm{~m}, 15 \mathrm{H}\left(\mathrm{C}_{6} \mathrm{H}_{3}\right) ; \\
7.91-8.13, \mathrm{~m}, 5 \mathrm{H}\left(\mathrm{OC}_{6} \mathrm{H}_{5}\right)\end{array}$ \\
\hline
\end{tabular}


A singlet at $\sim \delta 5.4 \mathrm{ppm}$ is due to the $\mathrm{CH}$ proton of benzoylacetonato moiety. Methyl tin protons give a singlet at $\sim \delta 1.62-1.85 \mathrm{ppm}$ and phenyl tin protons give a multiplet at $\sim \delta$ 7.4-7.8 ppm. An overlapping of the multiplets has been observed due to tin -methyl protons with the resonance signals of benzoylacetonato moiety.

${ }^{31} \mathrm{P}:$ Only one resonance signal in the region at $\delta 94.53$ to $98.82 \mathrm{ppm}$ is observed in the ${ }^{31} \mathrm{P}$ chemical shift values for these complexes (Table IV). On comparing these values with the parent compound, a downfield shift is observed. This unexpected downfield shift indicates that there is an isomerisation of S-alkyl trithiophosphate to O-alkyl trithiophosphate in these complexes and also indicates that Sn-S-P bond is formed in these complexes.

${ }^{119} \mathrm{Sn}:{ }^{1} \mathrm{H}$ and ${ }^{31} \mathrm{P}$ NMR spectral results of these complexes show the presence of single species, but ${ }^{119} \mathrm{Sn}$ NMR spectral results for these complexes [benzoylacetonato diorganotin(lV)-O-alkyl trithiophosphates] show the presence of three species(Table-IV). A comparison of these values with those of corresponding $\mathrm{R}_{2} \mathrm{Sn}[\mathrm{PhCOCHCOMe}]_{2}$ and $\mathrm{R}_{2} \mathrm{Sn}\left[\mathrm{SSH}(\mathrm{S}) \mathrm{POR}^{\prime}\right]_{2}$ compounds indicates that these complexes are also formed with mixed derivative, $\mathrm{R}_{2} \mathrm{Sn}[\mathrm{PhCOCHCOMe}]\left[\mathrm{SSH}(\mathrm{S}) \mathrm{POR}^{\prime}\right]$.

Table IV

${ }^{31} \mathrm{P}$ and ${ }^{119} \mathrm{Sn}$ NMR Spectral Data of Benzoylacetonato Diorganotin (IV)-O- Alkyl Trithiophosphate

\begin{tabular}{|c|c|c|c|c|c|}
\hline \multirow[t]{2}{*}{ S.N. } & \multirow[t]{2}{*}{ Compound } & \multirow[t]{2}{*}{${ }^{31} \mathbf{P}$ NMR } & \multicolumn{3}{|c|}{${ }^{119}$ Sn NMR } \\
\hline & & & I & II & III \\
\hline 1. & $\mathrm{Me}_{2} \mathrm{Sn}[\mathrm{SSH}(\mathrm{S}) \mathrm{POMe}][\mathrm{PhCOCHCOMe}]$ & 94.53 & -173.1 & -249.1 & -374.8 \\
\hline 2. & $\mathrm{Me}_{2} \mathrm{Sn}[\mathrm{SSH}(\mathrm{S}) \mathrm{POEt}][\mathrm{PhCOCHCOMe}]$ & 94.73 & -168.2 & -239.3 & -367.1 \\
\hline 3. & $\mathrm{Me}_{2} \mathrm{Sn}\left[\mathrm{SSH}(\mathrm{S}) \mathrm{POPr}^{\mathrm{i}}\right][\mathrm{PhCOCHCOMe}]$ & 95.21 & -157.5 & -235.4 & -361.4 \\
\hline 4. & $\mathrm{Me}_{2} \mathrm{Sn}[\mathrm{SSH}(\mathrm{S}) \mathrm{POBu}][\mathrm{PhCOCHCOMe}]$ & 96.44 & -175.1 & -251.7 & -370.0 \\
\hline 5. & $\mathrm{Me}_{2} \mathrm{Sn}[\mathrm{SSH}(\mathrm{S}) \mathrm{POPh}][\mathrm{PhCOCHCOMe}]$ & 97.32 & -182.4 & -280.8 & -399.2 \\
\hline 6. & $\mathrm{Bu}_{2} \mathrm{Sn}[\mathrm{SSH}(\mathrm{S}) \mathrm{POMe}][\mathrm{PhCOCHCOMe}]$ & 94.78 & -176.1 & -251.3 & -377.1 \\
\hline 7. & $\mathrm{Bu}_{2} \mathrm{Sn}[\mathrm{SSH}(\mathrm{S}) \mathrm{POEt}][\mathrm{PhCOCHCOMe}]$ & 95.22 & -170.3 & -242.2 & -368.5 \\
\hline 8. & $\mathrm{Bu}_{2} \mathrm{Sn}[\mathrm{SSH}(\mathrm{S}) \mathrm{POPr}][\mathrm{PhCOCHCOMe}]$ & 95.85 & -161.2 & -233.4 & -364.1 \\
\hline 9. & $\mathrm{Bu}_{2} \mathrm{Sn}\left[\mathrm{SSH}(\mathrm{S}) \mathrm{POBu}^{i}\right][\mathrm{PhCOCHCOMe}]$ & 96.74 & -178.7 & -254.2 & -372.2 \\
\hline 10. & $\mathrm{Bu}_{2} \mathrm{Sn}[\mathrm{SSH}(\mathrm{S}) \mathrm{POPh}][\mathrm{PhCOCHCOMe}]$ & 97.48 & -184.4 & -282.7 & -390.6 \\
\hline 11. & $\mathrm{Ph}_{2} \mathrm{Sn}[\mathrm{SSH}(\mathrm{S}) \mathrm{POMe}][\mathrm{PhCOCHCOMe}]$ & 95.94 & -177.2 & -252.1 & -378.2 \\
\hline 12. & 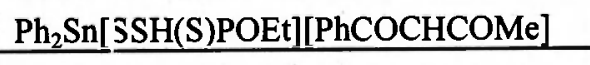 & 96.17 & -171.3 & -244.5 & -370.4 \\
\hline 13. & $\mathrm{Ph}_{2} \mathrm{Sn}\left[\mathrm{SSH}(\mathrm{S}) \mathrm{POPr}^{\mathrm{i}}\right][\mathrm{PhCOCHCOMe}]$ & 96.66 & -164.1 & -236.6 & -367.1 \\
\hline 14. & $\mathrm{Ph}_{2} \mathrm{Sn}\left[\mathrm{SSH}(\mathrm{S}) \mathrm{POBu}{ }^{1}\right][\mathrm{PhCOCHCOMe}]$ & 97.25 & -179.3 & -258.3 & -375.3 \\
\hline 15. & $\mathrm{Ph}_{2} \mathrm{Sn}[\mathrm{SSH}(\mathrm{S}) \mathrm{POPh}][\mathrm{PhCOCHCOMe}]$ & 98.82 & -182.5 & -284.4 & -395.8 \\
\hline
\end{tabular}

* The I and III resonance peaks correspond to $\mathrm{R}_{2} \mathrm{Sn}\left[\mathrm{SSH}(\mathrm{S}) \mathrm{POR}^{\prime}\right]_{2}$ and $\mathrm{R}_{2} \mathrm{Sn}[\mathrm{PhCOCHCOMe}]_{2}$, respectively. 
These spectral results indicate that as a result of disproportionation, the following equilibrium is obtained between these derivatives.

\section{$2 \mathrm{R}_{2} \mathrm{Sn}[\mathrm{PhCOCHCOMe}]\left[\mathrm{SSH}(\mathrm{S}) \mathrm{POR}^{\prime}\right] \quad \mathrm{N}_{2} \mathrm{Jn}[\mathrm{PhCOCHCOMe}]_{2}+\mathrm{R}_{2} \mathrm{Sn}\left[\mathrm{SSH}(\mathrm{S}) \mathrm{POR}^{\prime}\right]_{2}$}

These results also indicate the hexacoordinated nature of tin atom in these complexes. On the basis of spectroscopic studies, following structure can be suggested for benzoylacetonato diorganotin(lV)-O-alkyl trithiophosphates.

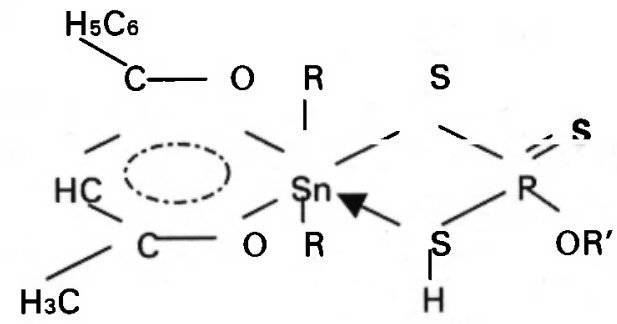

\section{ANTIBACTERIAL ACTIVITY}

All the newly synthesized compounds were screened for their antibacterial activity against gram-negative and gram-positive bacteria(Table-V).The activity was carried out by using the paper disc method. The zone of inhibition was measured in mm. DMF was used as a solvent. The compounds were tested at $500 \mu \mathrm{g} / \mathrm{mL}$ concentration. The observation shows that compounds $10,13,14$ are more effective against gram-negative bacteria and compounds 5,11,12 are more effective against gram-positive bacteria.

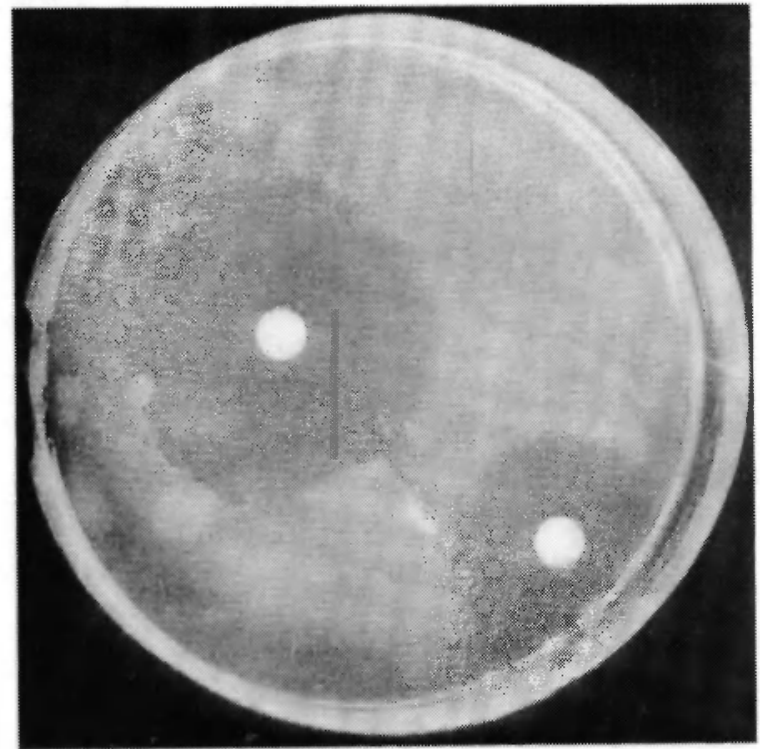

$\mathrm{Ph}_{2} \mathrm{Sn}[\mathrm{PhCOCHCOMe}]\left[\mathrm{SSH}(\mathrm{S}) \mathrm{POPr}^{\mathrm{i}}\right]$

effective against gram negative bacteria

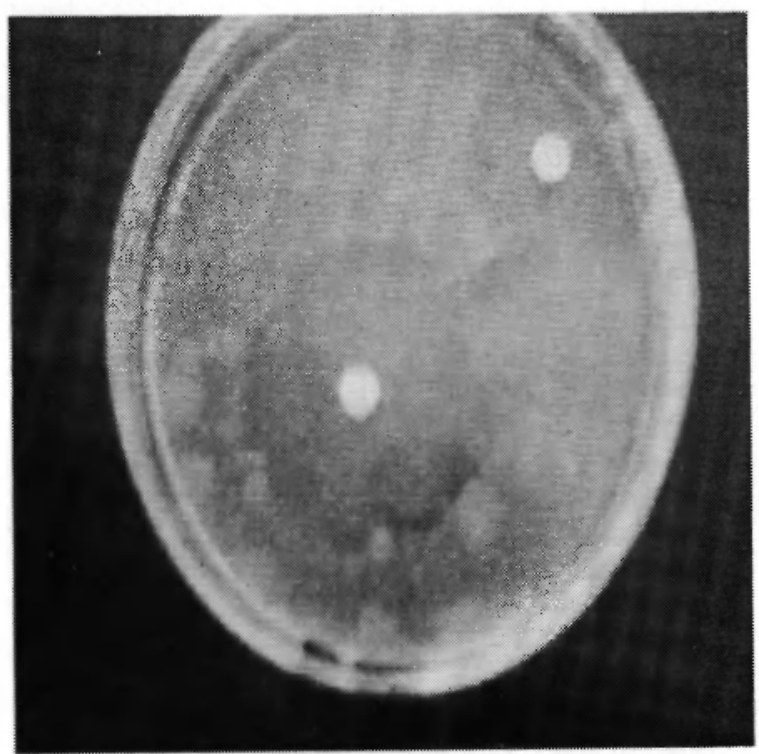

$\mathrm{Bu}_{2} \mathrm{Sn}[\mathrm{PhCOCHCOMe}][\mathrm{SSH}(\mathrm{S}) \mathrm{POPh}]$

effective against gram positive bacteria 
Table V

Antibacterial Activity of Benzoylacetonato Diorganotin (IV)-O-Alkyl Trithiophosphates [Zone Of Inhibition Measured In Mm]

\begin{tabular}{|c|c|c|c|}
\hline S.No. & Compounds & $\begin{array}{c}\text { Gram positive } \\
\text { bacteria }\end{array}$ & $\begin{array}{c}\text { Gram negative } \\
\text { bacteria }\end{array}$ \\
\hline 1. & $\mathrm{Me}_{2} \mathrm{Sn}[\mathrm{SSH}(\mathrm{S}) \mathrm{POMe}[\mathrm{PhCOCHCOMe}]$ & 8 & 28 \\
\hline 2. & $\mathrm{Me}_{2} \mathrm{Sn}[\mathrm{SSH}(\mathrm{S}) \mathrm{POEt}][\mathrm{PhCOCHCOMe}]$ & 10 & 31 \\
\hline 3. & $\mathrm{Me}_{2} \mathrm{Sn}\left[\mathrm{SSH}(\mathrm{S}) \mathrm{POPr}^{\mathrm{i}}\right][\mathrm{PhCOCHCOMe}]$ & 15 & 27 \\
\hline 4. & $\mathrm{Me}_{2} \mathrm{Sn}\left[\mathrm{SSH}(\mathrm{S}) \mathrm{POBu}^{1}\right][\mathrm{PhCOCHCOMe}]$ & 12 & 33 \\
\hline 5. & $\mathrm{Me}_{2} \mathrm{Sn}[\mathrm{SSH}(\mathrm{S}) \mathrm{POPh}][\mathrm{PhCOCHCOMe}]$ & 27 & 29 \\
\hline 6. & $\mathrm{Bu}_{2} \mathrm{Sn}[\mathrm{SSH}(\mathrm{S}) \mathrm{POMe}][\mathrm{PhCOCHCOMe}]$ & 11 & 32 \\
\hline 7. & $\mathrm{Bu}_{2} \mathrm{Sn}[\mathrm{SSH}(\mathrm{S}) \mathrm{POEt}][\mathrm{PhCOCHCOMel}$ & 20 & 29 \\
\hline 8. & $\mathrm{Bu}_{2} \mathrm{Sn}\left[\mathrm{SSH}(\mathrm{S}) \mathrm{POPr}^{\mathrm{i}}\right][\mathrm{PhCOCHCOMe}]$ & 23 & 37 \\
\hline 9. & $\mathrm{Bu}_{2} \mathrm{Sn}\left[\mathrm{SSH}(\mathrm{S}) \mathrm{POBu}^{\mathrm{i}}\right][\mathrm{PhCOCHCOMe}]$ & 18 & 40 \\
\hline 10. & $\mathrm{Bu}_{2} \mathrm{Sn}[\mathrm{SSH}(\mathrm{S}) \mathrm{POPh}][\mathrm{PhCOCHCOMe}]$ & 22 & 43 \\
\hline 11. & $\mathrm{Ph}_{2} \mathrm{Sn}[\mathrm{SSH}(\mathrm{S}) \mathrm{POMe}][\mathrm{PhCOCHCOMe}]$ & 29 & 35 \\
\hline 12. & $\mathrm{Ph}_{2} \mathrm{Sn}[\mathrm{SSH}(\mathrm{S}) \mathrm{POEt}[[\mathrm{PhCOCHCOMel}$ & 28 & 38 \\
\hline 13. & $\mathrm{Ph}_{2} \mathrm{Sn}\left[\mathrm{SSH}(\mathrm{S}) \mathrm{POPr}^{\mathrm{i}}\right][\mathrm{PhCOCHCOMe}]$ & 25 & 46 \\
\hline 14. & $\mathrm{Ph}_{2} \mathrm{Sn}\left[\mathrm{SSH}(\mathrm{S}) \mathrm{POBu}^{\prime}\right][\mathrm{PhCOCHCOMe}]$ & 15 & 41 \\
\hline 15. & $\mathrm{Ph}_{2} \mathrm{Sn}[\mathrm{SSH}(\mathrm{S}) \mathrm{POPh}][\mathrm{PhCOCHCOMe}]$ & 11 & 32 \\
\hline
\end{tabular}

\section{ACKNOWLEDGEMENT}

One of the authors (Leena Chordia) is thankful to C.S.I.R., New Delhi for financial assistance as J.R.F., S.A.I.F.(C.D.R.I.), Lucknow for spectral analysis and Dr. Mamta Goyal, Reader, Department of Botany, Government College, Ajmer for providing antibacterial screening facility.

\section{REFERENCES}

1. A. Chaturvedi, P.N. Nagar and G.Srivastava, Phosphorus, Sulfur and Silicon, 70, 303 (1992).

2. A. Chaturvedi, P.N. Nagar and G. Srivastava, Main Group Met. Chem., 16 (1), 45 (1993).

3. A. Chaturvedi, P.N. Nagar and G. Srivastava, Main Group Met. Chem., 16 (1), 53 (1993).

4. A. Chaturvedi, P.N. Nagar and G. Srivastava, Biol. Soc. Chil. Quim., 38, 265 (1993).

5. C. Gledal, J.P. Audieve, R. Clement and R. Cortes, J.Phys. Chim. Biol., 86 (7,8), 1691 (1989).

6. R.C. Mehrotra, Pure \& App. Chim., 60, 1349 (1988). 
7. A.D. Christle, R.A. Howle \& W. Moser, Inorg. Chim. Acta, 36, L 447 (1979) .

8. C.D. Chandler. G.D. Fallon, A.J. Koplick and B.O. West, Aust. J. Chem., 40, 1427 (1987).

9. S. Gopinathan, C. Gopinathan and J. Gupta, Indian J. Chem., 12, 626 (1974), D. Searle, P.J. Smith, N.A. Bell, I.A. March, I Nowell and J.D. Donaldson, Inorg. Chim. Acta., 162,143 (1989).

10. E.N.Vasanta. G. Srivastava and R.C. Mehrotra, Inorg. Chim. Acta., 30, 23 (1978).

11. S. Sharma, R. Bohra and R.C. Mehrotra, Synth. React. Inorg. Met.-Org. Chem., 21 (5), 741 (1991).

12. U.N. Tripathi and D.K. Sharma, Main Group Met. Chem., 27 (63), 135-144 (2004).

13. U.N. Tripathi and D.K.Sharma, Phosphorus, Sulfur and Silicon, 180, 1559 (2005).

14. A.I. Vogel "A Textbook of quantitative inorganic analysis", ELBS, IV Edition. London (1973) 
